\title{
Reduction of CCD observations of visual binaries using the "Tepui" function as PSF`
}

\author{
C. Abad ${ }^{1}$, J. A. Docobo ${ }^{2}$, V. Lanchares ${ }^{3}$, J. F. Lahulla ${ }^{4}$, P. Abelleira ${ }^{2}$, J. Blanco ${ }^{2}$, and C. Alvarez ${ }^{2}$ \\ 1 Centro de Investigaciones de Astronomía, CIDA, 5101-A Mérida, Venezuela \\ 2 Observatorio Astronómico Ramón María Aller, Universidade de Santiago de Compostela, PO Box 197, \\ Santiago de Compostela, Spain \\ 3 Departamento de Matemáticas y Computación, Universidad de La Rioja, c./ Luis de Ulloa s/n, Logroño, Spain \\ 4 Observatorio Astronomico Nacional, OAN, c./ Alfonso XII, 3, Madrid, Spain
}

Received 19 March 2003 / Accepted 19 September 2003

\begin{abstract}
CCD measurements of relative positions and magnitude differences in $V$ and $R$ photometric bands for 165 visual double and multiple stars are given. CCD frames were taken at the $1.52 \mathrm{~m}$ Spanish telescope of the Spanish-German Center of Astronomy at Calar Alto (Almería, Spain). During the reduction process a "Tepui" function was used as the PSF function.
\end{abstract}

Key words. stars: general - astrometry - techniques: photometric

\section{Introduction}

The use of CCDs for observations of wide and not very close visual double stars has steadily displaced conventional techniques - visual measurements - almost in a definitive way. The CCD's geometrical stability, the wide dynamic range, the linear response and the development of techniques for the data analysis are some reasons to feel confident about the accuracy of resulting positional and photometric measurements.

Conventional programs such as IRAF or MIDAS have the necessary software packages to leave frames in optimal conditions. They make use of the calibration frames (bias, flats, darks) in an easy-to-use form.

The angular separation of the double star observed defines the reduction. Most people look for the point spread functions (PSF), if possible. If the frame contains other images or the pair is of wide separation, the PSF is obtained from the best-defined stellar images (Sinachopoulos \& Seggewis 1990). However, if the double star is alone and image overlapping exists, an iterative process can be applied (Tokovinin \& Shatskii 1995).

Since 1994, CIDA has been collaborating with the Astronomical Observatory Ramón María Aller in a program of observations of southern visual double stars, with CCD and micrometric techniques from the Venezuelan National Observatory (Abad et al. 1998). Each observation and reduction program was usually carried out by the Spanish or Venezuelan institution in an independent way. In this paper,

Send offprint requests to: C. Abad, e-mail: abad@cida.ve

* Tables 1 and 2 are only available in electronic form at the CDS via anonymous ftp to cdsarc.u-strasbg. fr (130.79.128.5) or via http://cdsweb.u-strasbg.fr/cgi-bin/qcat?J/A+A/416/811 observations have been carried out with $1.52 \mathrm{~m}$ Spanish telescope at the Spanish-German Center of Astronomy at Calar Alto (Almería, Spain). The Venezuelan contribution has been the introduction of the fitting function (Abad 1996), called the Tepui function, for the reduction of the observations. This function was created for astrometric purposes, reproducing successful image profiles, especially for those saturated.

The advantage of the use of this function is its adaptability to the real profile of the images, much better than the traditional Gaussian or similar functions. The fitting function is necessary to obtain the magnitude difference for overlapping components. We suppose that this PSF exists for a whole frame and optical, instrumental and seeing differences between images are not significant, especially for components of the pair.

\section{Observations}

The $1.52 \mathrm{~m}$ National Astronomical Observatory (OAN) telescope used for the observations is equipped with a TK1024 AB chip with $1024 \times 924$ pixels, each of 24 micron square. Two Johnson filters were used:

V BG $18(2 \mathrm{~mm})+\mathrm{GC} 495(2 \mathrm{~mm})$

R OG $570(2 \mathrm{~mm})+\mathrm{KG} 3(2 \mathrm{~mm})$.

A total of 400 frames were obtained and reduced for this paper, containing information on about 165 double and multiple stars of the initial observational program. Most of them have one or several observations in $R$ and $V$ bands.

Observations were carried out in July and August 1998, and calibration frames (bias, dark and flat frames) were also taken. 
The exposure time depends on the system brightness but on average it was about $20 \mathrm{~s}$. Seeing conditions were moderately favorable, from about 1". 4 to 2 '. 0 with most of them near 1'. 6 .

Dark counts may be neglected since they were very weak. See the CCD characteristics on the OAN web page, Calar Alto Instrumentation Section (http://pcweb.oan.es/1.52m/ ccd.html)

\section{The Tepui function}

A critical step for the reduction of the observations with good astrometric quality is the measurement of the $(x, y)$ rectangular coordinates of the images from a plate or frame. To resolve this problem, and those caused by image saturation, the astrometric section at CIDA developped the Tepui function which can reproduce more accurately than others the real profile of not too faint images. An example of this can be found in Abad \& Vicente (1999).

This function is based on the difference between two arctangent functions with a general analytical expression for a bidimensional case:

$y=A[\operatorname{arctg}(b(x-c))-\operatorname{arctg}(b(x+c))]$

where:

$A$ is the amplitude after applying a normalization factor;

$b$ is related to the tilt of the profile of the image;

$c$ is related to the width of the profile of the image.

Two important characteristics can be pointed out from this function. First, due to construction (Fig. 1), in a process of fitting by the least square method, the image's lateral data are the most important for the reproduction of the real profile of the image. It is different when the fitting function is a Gaussian, where central points control the process. Second, if we suppose that the natural light distribution over the image follows a normal distribution, no considerable differences are introduced when we fit a tepui distribution (Fig. 2).

In this paper we want to introduce this function to obtain relative positions and magnitude differences of stellar pairs.

The advantage of this method is based on the fact that many stellar images in the field have not always available to get a reliable PSF function and, in addition, there exists overlap of the image components. We present a function that can reproduce the real profile of the image.

When saturation problems appear, relative positions can be obtained from this fitting function, but, obviously, not magnitude differences in a direct form. Nevertheless we hope that it will become possible to reconstruct the top of the image fitting values into the linear response of the CCD with the Tepui function.

\section{Reduction of observations}

Facilities from IRAF have been used to apply the calibration frames to program frames, subtracting bias to flat and program frames, normalizing the resulting flat frame and applying this to the resulting program frames. Next, a square area containing

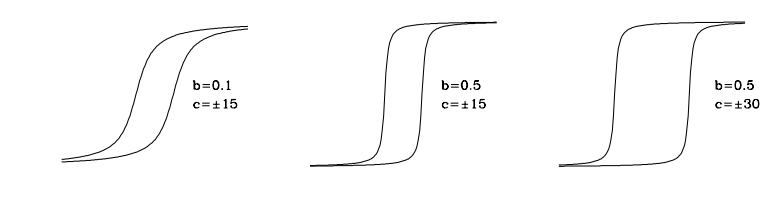

Original pairs of arctangent functions for diferent $\mathrm{b}$ and $\mathrm{c}$ parameters
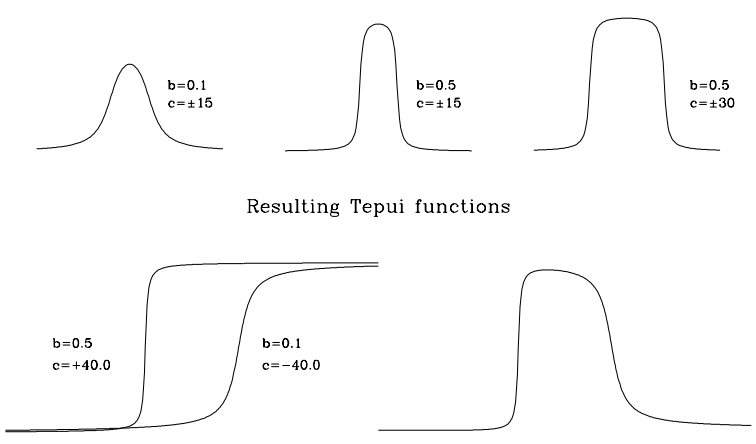

Generating an asymmetric Tepui function

Fig. 1. Upper diagram: graphic description of the construction of symmetric and asymmetric "tepui functions" from a pair of arctangent functions.

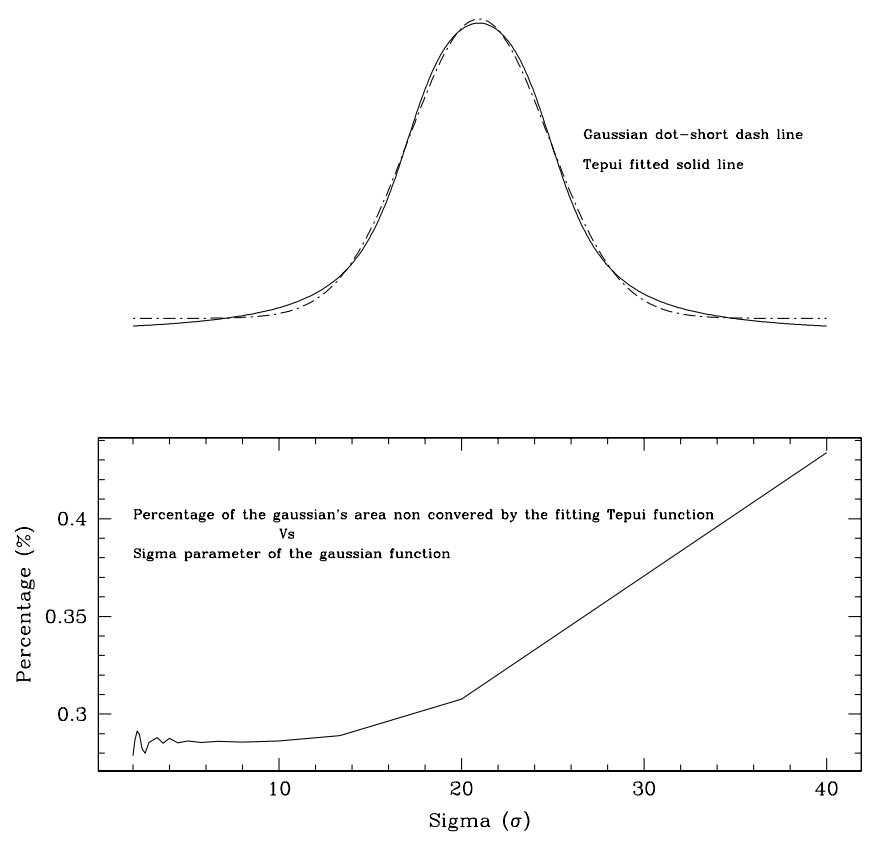

Fig. 2. Upper diagram: Tepui function reproduces closely the Gaussian function. Lower diagram: percentage of the Gaussian's area no-covered by the fitting tepui function vs. the width of the Gaussian function. This width can represent the FWHM of the image. The representation is independent of the amplitude of the Gaussian function.

the pair is isolated to get the relative position between components as the relative position between the two fitting functions applied to their images. In this paper we use the Tepui function as the fitting function. This function in a tridimensional space has a general analytical expression as:

$f(u, v)=C_{1} \times C_{2} \times C_{3}+C_{4}$ 
where:

$C_{1}=\frac{A}{4 \operatorname{arctg}\left(b_{\mathrm{u}} c_{\mathrm{u}}\right) \operatorname{arctg}\left(b_{\mathrm{v}} c_{\mathrm{v}}\right)}$

$C_{2}=\left[\operatorname{arctg}\left(b_{\mathrm{u}}\left(u-c_{\mathrm{u}}\right)\right)-\operatorname{arctg}\left(b_{\mathrm{u}}\left(u+c_{\mathrm{u}}\right)\right)\right]$

$C_{3}=\left[\operatorname{arctg}\left(b_{\mathrm{v}}\left(v-c_{\mathrm{v}}\right)\right)-\operatorname{arctg}\left(b_{\mathrm{v}}\left(v+c_{\mathrm{v}}\right)\right)\right]$

$C_{4}=\zeta_{1}+\zeta_{1} u+\zeta_{1} v$

$u=\left(x-x_{0}\right) \cos (\phi)+\left(y-y_{0}\right) \sin (\phi)$

$v=-\left(x-x_{0}\right) \sin (\phi)+\left(y-y_{0}\right) \cos (\phi)$

and the meaning of each parameter is:

$A$ amplitude;

$\left(b_{\mathrm{u}}, b_{\mathrm{v}}\right)$ are related to the tilt of the profile of the image for each axis;

$\left(c_{\mathrm{u}}, c_{\mathrm{v}}\right)$ are related to the width of the profile of the image for each axis;

$\left(x_{0}, y_{0}\right)$ rectangular coordinates of the center of the image;

$\phi$ angle of orientation of the image with respect to the CCD orientation;

$C_{4}$ plane fitting of the background of the image.

For a double star we need to use a double fitting function composed of the sum of two simple functions with a common width $\left(c_{\mathrm{u}}\right.$ and $c_{\mathrm{v}}$ parameters), similar orientation $(\phi)$ and the same base $\left(C_{4}\right)$. The center of symmetry of the primary star $\left(x_{0}, y_{0}\right)$ is transformed into the origin of coordinates during the process and the relative position between functions $(\mathrm{d} x, \mathrm{~d} y)$ will be the center of symmetry of the secondary star.

Sixteen independent parameters must be determined. Three of them correspond to the background fit.

It is possible to consider total asymmetry with the Tepui function taken as independent the tilt parameter on each side of the bi-dimensional Tepui function. This adds two more parameters. We consider this not necessary and perform the process with a double-axis symmetry.

Angular scales were obtained astrometrically, reducing by Stock's method (Stock 1981) those crowed frames and getting the scale factor of the optical system of the telescope. We obtained a scale of $0 . ' 465 \pm 0$. '002 per pixel.

The orientation of the CCD was calculated from the streaks in non-guided observations. A small correction of $0.7^{\circ} \pm 0.001$ must be applied.

Differences of magnitudes were obtained as quotient of volumes included under both fitting function as:

$\Delta m=-2.5+\log \left(\frac{\Phi_{A}}{\Phi_{B}}\right)$

where $\Phi$ is the volume or flux.

Results were compared with those obtained by members of the collaboration with the $1.23 \mathrm{~m}$ German telescope at the same Astronomical Center (Docobo et al. 2000), obtained with similar standard techniques, when stars of both observational programs coincide. An excellent agreement exists.

Checks were also performed using crowded frames. Common parameters, relative to width and orientation of the
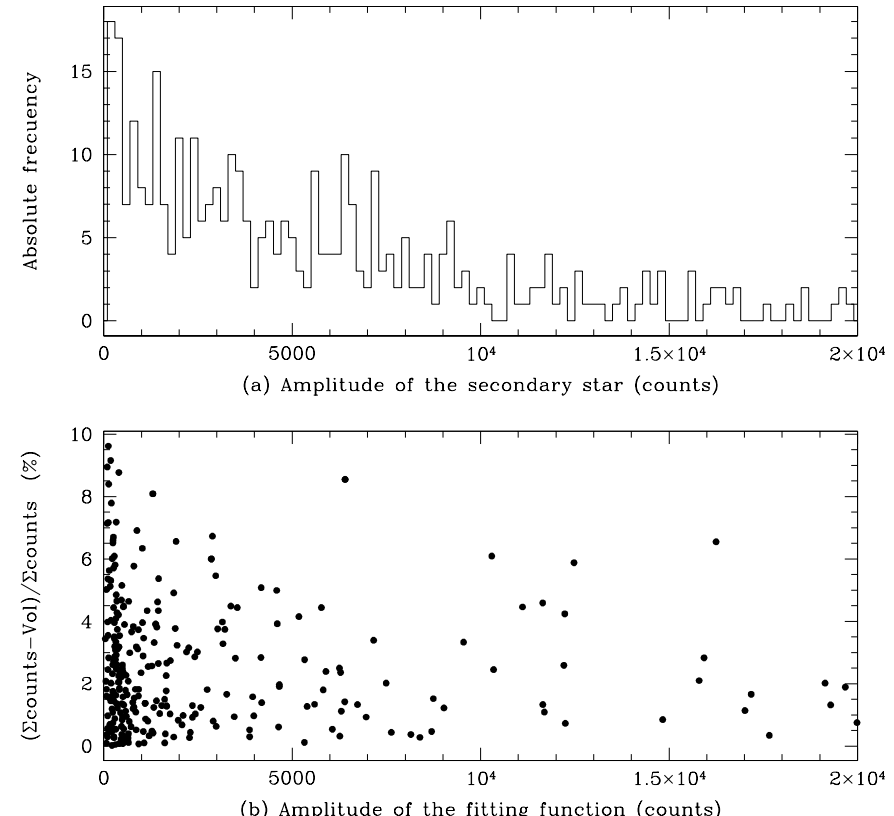

Fig. 3. Upper diagram: histogram of amplitudes of secondary stars of the double star program. Lower diagram: percentage of counts covered by the Tepui function versus the amplitude of the image fitted. Vol. is the volume under the Tepui function.

double star, remain constant during the fitting of the individual Tepui function to other images of the frame. The noncoincidence of fitting function and image, over a selected area that encloses the image, determined as the rate of the function's integral minus the total counts over the total counts, can give us an estimation of the confidence of the Tepui function for these purposes.

The upper diagram of Fig. 3 shows the histogram of amplitudes of secondary stars of the double star program. The lower diagram shows the rate expressed as percentage versus the amplitude of the image fitted.

A higher dispersion of percentages was found for observations made with the $R$ filter than $V$ filter as show Fig. 4 .

This percentage never exceeds $10 \%$ and most of them are around two or three per cent. This supposes that the typical error in the determination of the difference of magnitudes is around 0 . 02 mag.

However, sometimes one frame contains other apparent doubles stars. In this case we reduce them giving their coordinates (J2000) extracted by astrometry reduction or given by the USNO-2 catalogue as a reference.

\section{Results}

Table 1 gives relevant data for the visual binaries observed. Observations were carried out over a short period of time, therefore we assign the mean epoch (1998.58) for the totality of data. Data of individual stars are presented as average data with errors, by parameter, when various observations have been calculated, as well as the number of observations involved. Errors corresponding to astrometric relative positions between components are always present. For single observations, parameter 


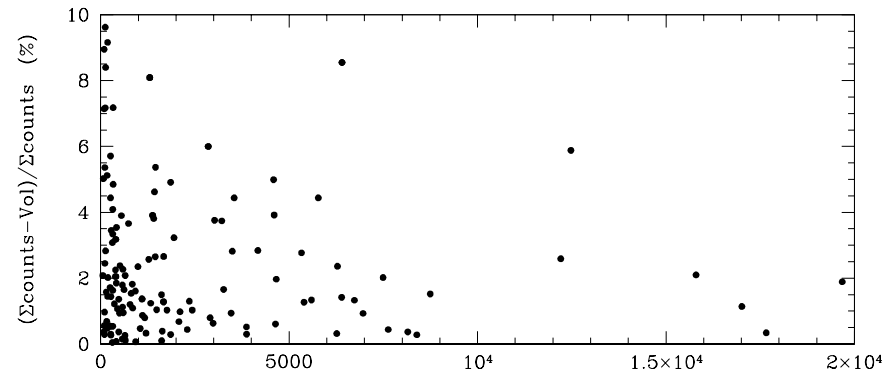

(a) Amplitude of the fitting function for $\mathrm{V}$ filter (counts)

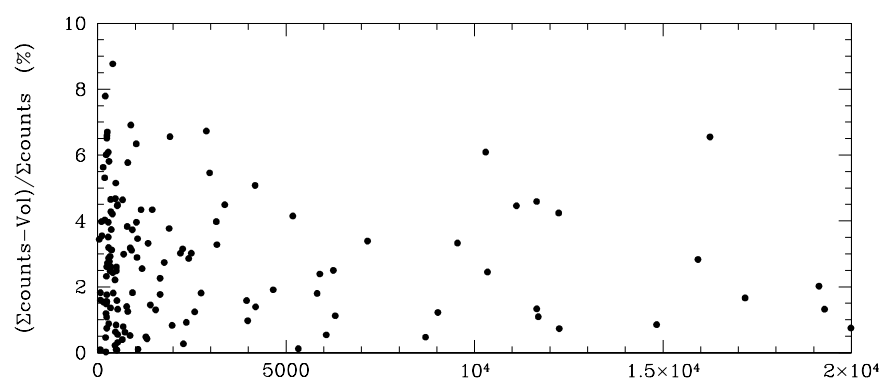

(b) Amplitude of the fitting function for $\mathrm{R}$ filter (counts)

Fig. 4. Percentage of counts covered by the Tepui function versus the amplitude of the image fitted but separated by filters. a) for $V$ filter. b) for $R$ filter. Vol is the volume under the Tepui function.

Table 1. Data for the visual binaries observed; only available in electronic form at the CDS.

Table 2. Data for unidentified visual binaries observed; only available in electronic form at the CDS.

fitting errors, specially for $\mathrm{d} x$ and $\mathrm{d} y$ parameters, have been calculated analysing the $\chi^{2}$ test around the minimum. Following the rules for error propagation, $\theta$ and $\rho$ errors can be estimated. Table 1 shows single observation errors with an additional significant digit.

When a star does not have known references, we include it in Table 2, where $\mathbf{J} 2000$ position and magnitudes are from the USNO-A2.0 catalogue (Monet et al. 1998).

Stars in the table are arranged in increasing RA in the following order:

WDS WDS catalogue number (Mason et al. 2003),

Name WDS catalogue name (Mason et al. 2003),

ADS the ADS number (Aiken 1932),

$\theta \quad$ Position Angle in degrees,

$\sigma_{\theta} \quad$ error of Position Angle,

$\rho \quad$ Separation of the components in arcsecs,

$\sigma_{\rho} \quad$ error of Separation,

$\Delta m$ difference of magnitude of the components,

$\sigma_{\Delta m}$ error of difference of magnitude,

$B$ filter used,

$n \quad$ number of observations.

Finally, Table 3 shows differences obtained by comparing data in this paper and those shown in Docobo et al. (2000). Columns 1 to 3 are similar to those in Tables 1 and 2 .
Table 3. Comparison between data from this paper $(\mathrm{T})$ and those obtained from standard (S) reduction in Docobo et al. (2000) for common stars. Residuals from this comparison $(\mathrm{T}-\mathrm{S})$ are given.

\begin{tabular}{|c|c|c|c|c|c|c|}
\hline WDS & Name & ADS & $\theta_{(\mathrm{T}-\mathrm{S})}$ & $\rho_{(\mathrm{T}-\mathrm{S})}$ & $\Delta m_{(\mathrm{T}-\mathrm{S})}$ & $B$ \\
\hline $00255+6129$ & STI $57 A B$ & 340 & $\begin{array}{l}0.5 \\
0.3\end{array}$ & $\begin{array}{l}0.02 \\
0.02\end{array}$ & $\begin{array}{r}-0.01 \\
0.00\end{array}$ & $\begin{array}{l}R \\
V\end{array}$ \\
\hline $00302+5929$ & STI 79 & 402 & $\begin{array}{l}-0.2 \\
-0.4\end{array}$ & $\begin{array}{l}0.03 \\
0.03\end{array}$ & $\begin{array}{l}0.02 \\
0.12\end{array}$ & $\begin{array}{l}R \\
V\end{array}$ \\
\hline $01419+4646$ & ES 1211 & 1330 & $\begin{array}{r}-0.3 \\
0.1\end{array}$ & $\begin{array}{r}-0.01 \\
0.05\end{array}$ & $\begin{array}{l}0.02 \\
0.00\end{array}$ & $\begin{array}{l}R \\
V\end{array}$ \\
\hline $\begin{array}{l}17372+3124 \\
17372+3124\end{array}$ & SEI 544 & $\begin{array}{l}10672 \\
10672\end{array}$ & $\begin{array}{l}0.2 \\
0.1\end{array}$ & $\begin{array}{l}0.02 \\
0.03\end{array}$ & $\begin{array}{l}-0.05 \\
-0.11\end{array}$ & $\begin{array}{l}R \\
V\end{array}$ \\
\hline $\begin{array}{l}17484+2942 \\
17484+2942\end{array}$ & BRT 30 & $\begin{array}{l}10811 \\
10811\end{array}$ & $\begin{array}{l}-0.2 \\
-0.8\end{array}$ & $\begin{array}{r}-0.00 \\
0.06\end{array}$ & $\begin{array}{l}0.03 \\
0.06\end{array}$ & $\begin{array}{l}R \\
V\end{array}$ \\
\hline $\begin{array}{l}21069+3845 \\
21069+3845\end{array}$ & $S T F 2758 A B$ & $\begin{array}{l}14636 \\
14636\end{array}$ & $\begin{array}{l}0.3 \\
0.3\end{array}$ & $\begin{array}{l}-0.12 \\
-0.00\end{array}$ & $\begin{array}{l}0.00 \\
0.01\end{array}$ & $\begin{array}{l}R \\
V\end{array}$ \\
\hline $\begin{array}{l}22026+6238 \\
22026+6238\end{array}$ & STI 1081 & $\begin{array}{l}15582 \\
15582\end{array}$ & $\begin{array}{l}0.2 \\
0.0\end{array}$ & $\begin{array}{l}0.00 \\
0.01\end{array}$ & $\begin{array}{l}0.00 \\
0.00\end{array}$ & $\begin{array}{l}R \\
V\end{array}$ \\
\hline
\end{tabular}

Columns 4-6 contain the relative position (orientation and distance) and magnitude difference for common stars.

Acknowledgements. This paper was financed by research projects AYA 2001-3073 and PGIDIT02 PXIC24301PN both directed by Prof. J.A. Docobo and supported by Spanish Ministerio de Ciencia y Tecnología and Xunta de Galicia respectively. The authors thank the Observatorio Astronómico Nacional (IGN) for all facilities provided during the observational run.

\section{References}

Abad, C. 1996, Ph.D. Thesis, Publicaciones del Seminario Matemático García de Galdeano, Ser. II (52), Universidad de Zaragoza, Zaragoza, Spain

Abad, C., Docobo, J. A., \& Della Prugna, F. 1998, A\&AS, 133, 71

Abad, C., \& Vicente, B. 1999, A\&AS, 136, 307

Aitken, R. G. 1932, New General Catalogue of Double Stars (ADS), Carnegie Institution of Washington, 417

Docobo, J. A., Alvarez, C., Lahulla, J. F., Lanchares, V., \& Aguirre, A. 2000, Astron. Nachr., 321, 53

Mason, B. D., Wycoff, G. L., \& Hartkopf, W. I. 2003, The Washington Visual Double Star Catalogue, Electronic version US Naval Observatory (http://ad.usno.navy. mil/wds/wds.html)

Monet, D., Bird, A., Canzian, B., et al. 1998, The USNOA2.0 Catalogue. VizieR On-line Data Catalog I/252 (http://vizier.u-strasbg.fr). Originally published in US Naval Observatory Flagstaff Station (USNOFS) and Universities Space Research Association (USRA) stationed at USNOFS

Sinachopoulos, D., \& Seggewis, W. 1990, A\&AS, 83, 245

Stock, J. 1981, Rev. Mex. Astron. Astrofís., 6, 115

Tokovinin, A. A., \& Shatskii, N. I. 1995, AZh, 21, 523 